\title{
A Generalized F-test for the Mean of A Class of Elliptically Contoured Distributions
}

\author{
Jiajuan Liang \\ College of Business, University of New Haven, West Haven, Connecticut, U.S.A. \\ Email: jliang@newhaven.edu
}

\begin{abstract}
The theory of spherical distributions is employed to develop a generalized F-test for testing the mean of a subfamily of elliptically contoured distributions. The exact null distribution of the generalized F-test is obtained. The power performance of the generalized F-test is illustrated by choosing several distributions in the subfamily of elliptically contoured distributions. The Monte Carlos study shows that the generalized F-test is not sensitive to the increase of sample dimension. The generalized F-test is applicable to the case of any dimension with any sample size. An analysis on a real dataset in financial models illustrates possible applications of the proposed tests.
\end{abstract}

Keywords: Elliptically contoured distribution; generalized F-test; high dimension; spherically symmetric distribution.

\section{Introduction}

The family of elliptically contoured distributions (ECD for simplicity) is a natural extension to the classical multivariate normal distribution $N_{p}(\boldsymbol{\mu}, \boldsymbol{\Sigma})$. Many theoretical results for ECD have been obtained since the last few decades ([2]). Following the same notation as in [2], we denote a $p$-dimensional distribution in the family of $\operatorname{ECD}$ by $\operatorname{ECD}(\boldsymbol{\mu}, \boldsymbol{\Sigma})$ with a mean vector $\boldsymbol{\mu}(p \times 1)$ and a covariance matrix $\boldsymbol{\Sigma} \geq \mathbf{0}(p \times p$, non-negative definite). It is well-known that a distribution in $\operatorname{ECD}(\boldsymbol{\mu}, \boldsymbol{\Sigma})$ may not have a probability density function. This makes it difficult to develop any parametric statistical inference on the mean vector $\boldsymbol{\mu}$ or the covariance matrix $\boldsymbol{\Sigma}$. Fang and Zhang (1990) ([2]) gave some thoughts on statistical inference on $\boldsymbol{\mu}$ and $\boldsymbol{\Sigma}$ based on the theory of spherical-matrix distributions, where the samples are not traditional ones with the i.i.d. (independently identically distributed) assumption. If an $\operatorname{ECD}(\boldsymbol{\mu}, \boldsymbol{\Sigma})$ has a probability density function, it must be of the form $f\left[(\boldsymbol{x}-\boldsymbol{\mu})^{\prime} \boldsymbol{\Sigma}^{-1}(\boldsymbol{x}-\boldsymbol{\mu})\right](\boldsymbol{\Sigma}$ must be positive definite $)$ with some nonnegative scalar function $f(\cdot)$. Even so, the the scalar function $f(\cdot)$ is still unknown. This makes it impossible to construct the traditional likelihood ratio test. The uncertainty of an ECD with or without a probability density function creates the major difficulty in developing parametric statistical inference on the two parameters $\boldsymbol{\mu}$ and $\boldsymbol{\Sigma}$.

Because of the great challenge in developing parametric statistical inference on the two parameters $\boldsymbol{\mu}$ and $\boldsymbol{\Sigma}$ for a general $\operatorname{ECD}(\boldsymbol{\mu}, \boldsymbol{\Sigma})$, we try to tackle this problem by considering a subfamily $E C D_{p}\left(\boldsymbol{\mu}, \sigma^{2} \boldsymbol{I}_{p}\right)$ with an i.i.d. sample, where $\sigma>0$ is unknown and $\boldsymbol{I}_{p}$ stands for the $p \times p$ identity matrix. Without loss of generality, testing the mean $\boldsymbol{\mu}$ in $E C D_{p}\left(\boldsymbol{\mu}, \sigma^{2} \boldsymbol{I}_{p}\right)$ can be reduced to testing the hypothesis

$$
H_{0}: \boldsymbol{\mu}=\mathbf{0}, \quad \text { versus } \quad H_{1}: \boldsymbol{\mu} \neq \mathbf{0} .
$$

We will develop the theoretical details for constructing the test for hypothesis (1) under an i.i.d. sample in section 2. Section 3 will present the Monte Carlo study on the performance of the test. Section 4 illustrates some possible application of the proposed test. Some concluding remarks are summarized in the last section.

\section{Theoretical Development}

The following theorem provides the theoretical basis for the generalized F-test. 
Theorem 1. Let $\left\{\boldsymbol{x}_{1}, \ldots, \boldsymbol{x}_{n}\right\}$ be an i.i.d. sample from $E C D_{p}\left(\boldsymbol{\mu}, \sigma^{2} \boldsymbol{I}_{p}\right)$. For each $\boldsymbol{x}_{i}=\left(x_{i 1}, \ldots, x_{i p}\right)^{\prime}$ $(p \times 1)$, define

$$
F_{i}\left(\boldsymbol{x}_{i}\right)=\left(\sqrt{p} \bar{x}_{i}\right)^{2} /\left[\frac{1}{p-1} \sum_{j=1}^{p}\left(x_{i j}-\bar{x}_{i}\right)^{2}\right]
$$

for $i=1, \ldots, n$, where $\bar{x}_{i}=(1 / p) \sum_{j=1}^{p} x_{i j}$ is sample mean from $\boldsymbol{x}_{i}=\left(x_{i 1}, \ldots, x_{i p}\right)^{\prime}$. Construct the generalized F-statistic by

$$
G F=\max _{1 \leq i \leq n}\left\{F_{i}\left(\boldsymbol{x}_{i}\right)\right\}
$$

Under the null hypothesis in (1), GF has an exact distribution given by

$$
P(G F<x)=[F(x ; 1, p-1)]^{n}, \quad x \geq 0,
$$

where $F(\cdot ; 1, p-1)$ stands for the cumulative distribution function of the F-distribution with degrees of freedom $(1, p-1)$.

Proof. Note that under the null hypothesis in (1), each observation $\boldsymbol{x}_{i}(i=1, \ldots, n)$ in the i.i.d. sample $\left\{\boldsymbol{x}_{1}, \ldots, \boldsymbol{x}_{n}\right\}$ has an $\operatorname{ECD}\left(\mathbf{0}, \sigma^{2} \boldsymbol{I}_{p}\right)$, which reduces to a spherically symmetric distribution [1]. Each statistic $F_{i}$ defined by $(2)$ is scale-invariant:

$$
F_{i}\left(a \boldsymbol{x}_{i}\right)=F_{i}\left(\boldsymbol{x}_{i}\right)
$$

for any constant $a>0$. According to [1], we have

$$
F_{i}\left(\boldsymbol{x}_{i}\right) \stackrel{d}{=} F_{i}\left(\boldsymbol{z}_{0}\right)
$$

where $\boldsymbol{z}_{0} \sim N_{p}\left(\mathbf{0}, \boldsymbol{I}_{p}\right)$, the $p$-dimensional standard normal distribution, and the notation " $\stackrel{d}{=}$ means that both sides of the equation have the same distribution. It is noted that

$$
F_{i}\left(\boldsymbol{z}_{0}\right)=[\sqrt{p} \bar{z}]^{2} /\left[\frac{1}{p-1} \sum_{j=1}^{p}\left(z_{j}-\bar{z}\right)^{2}\right] \stackrel{d}{=}[t(p-1)]^{2} \stackrel{d}{=} F(1, p-1)
$$

where $\boldsymbol{z}_{0}=\left(z_{1}, \ldots, z_{p}\right)^{\prime}, \bar{z}=(1 / p) \sum_{j=1}^{p} z_{j}$, and $t(p-1)$ stands for the Student's t-distribution with degrees of freedom $p-1$. The assumption on the i.i.d. sample leads to:

$$
\begin{aligned}
& P(G F<x)=P\left(\max _{1 \leq i \leq n}\left\{F_{i}\left(\boldsymbol{x}_{i}\right)\right\}<x\right)=P\left(\cap_{i=1}^{n}\left\{F_{i}\left(\boldsymbol{x}_{i}\right)<x\right\}\right) \\
= & \prod_{i=1}^{n} P\left(F_{i}\left(\boldsymbol{x}_{i}\right)<x\right)=[F(x ; 1, p-1)]^{n}, \quad x>0 .
\end{aligned}
$$

This completes the proof.

The GF-statistic in (3) can be applied to test hypothesis (1). If the null hypothesis in (1) is true, GF tends to departure from zero. The critical value $c(\alpha)$ at a given level $0<\alpha<1$ for the $G F$-test can be accurately determined by

$$
\begin{aligned}
& P(G F>c(\alpha))=1-P(G F<c(\alpha))=1-[F(c(\alpha) ; 1, p-1)]^{n}, \\
& \text { or } c(\alpha)=F_{\text {inv }}\left((1-\alpha)^{\frac{1}{n}} ; 1, p-1\right),
\end{aligned}
$$

where $F_{\text {inv }}(\cdot ; 1, p-1)$ stands for the inverse of the cumulative distribution function of the F-distribution $F(1, p-1)$. Computer programs for Statistical computation such as MATLAB usually provides the computation for $F_{\text {inv }}(\cdot ; 1, p-1)$. The principle for testing hypothesis $(1)$ by the generalized F-test $G F$-statistic can be summarized as: for a given level $0<\alpha<1$, reject the null hypothesis if $G F>c(\alpha)$ with $c(\alpha)$ computed by (8). 


\section{A Monte Carlo Study}

The following subfamilies of $E C D_{p}\left(\boldsymbol{\mu}, \boldsymbol{I}_{p}\right)(\sigma=1)$ (Chapter 3 in [1]) are chosen for simulating the empirical type I error rates and the empirical power by using MATLAB code, where the $C_{i}(i=1,2,3,4,5)$ are suitable normalizing constants.

(1) The multivariate normal distribution $N_{p}\left(\boldsymbol{\mu}, \boldsymbol{I}_{p}\right)$;

(2) The multivariate t-distribution has a density function of the form

$$
f_{t}(\|\boldsymbol{x}-\boldsymbol{\mu}\|)=C_{1}\left(1+\frac{\|\boldsymbol{x}-\boldsymbol{\mu}\|^{2}}{m}\right)^{-\frac{p+m}{2}}, \quad m>0
$$

where " $\|\cdot\| "$ stands for the Euclidean norm of a vector, let $m=5$;

(3) the $\operatorname{Kotz}$ type distribution $\operatorname{Kotz}(N, m, s)$ with parameters $N=2, m=1$ and $s=0.5$ has a density function of the form

$$
f_{k}(\|\boldsymbol{x}-\boldsymbol{\mu}\|)=C_{2}\|\boldsymbol{x}-\boldsymbol{\mu}\|^{2(N-1)} \exp \left\{-r\|\boldsymbol{x}-\boldsymbol{\mu}\|^{2 s}\right\}
$$

where $N, r$ and $s$ are parameters. Let $N=2, r=1$ and $s=0.5$.

(4) The Pearson type II distribution with the density function of the form

$$
f_{P 2}(\|\boldsymbol{x}-\boldsymbol{\mu}\|)=C_{3}\left(1-\|\boldsymbol{x}-\boldsymbol{\mu}\|^{2}\right)^{-m},
$$

where $m>-1$ is a parameter. Let $m=3 / 2$.

(5) The Pearson type VII distribution with the density function of the form

$$
f_{P 7}(\|\boldsymbol{x}-\boldsymbol{\mu}\|)=C_{4}\left(1+\frac{\|\boldsymbol{x}-\boldsymbol{\mu}\|^{2}}{m}\right)^{-N}, \quad N>p / 2, m>0
$$

Let $m=2$ and $N=20$ for $p=10$ and $N=30$ for $p=50$.

(6) The multivariate Cauchy distribution has a density function of the form

$$
f_{\text {Cauchy }}(\|\boldsymbol{x}-\boldsymbol{\mu}\|)=C_{5}\left(1+\|\boldsymbol{x}-\boldsymbol{\mu}\|^{2}\right)^{-\frac{p+1}{2}} .
$$

An i.i.d. sample from each of these distributions can be easily generated by MATLAB code (available from the authors upon request). Without loss of generosity, the mean vector $\boldsymbol{\mu}$ is chosen as $\boldsymbol{\mu}=c \mathbf{1}_{p}$ and let the constant $c$ increase from $c=0$ to a suitable number until the power can reach 1 approximately with a suitable equal increment, which means that taking the equal step length. The power is computed by taking the significance level $\alpha=0.05$. In order to compare the impact of the sample size $n$ on the power performance, we plot the power values versus the $c$-values. Figure 1 presents the plots of power values against the departure from zero of the mean vector $\boldsymbol{\mu}=c \mathbf{1}_{p}$ with increasing $c$-values for relatively low dimensions $p=5$ and $p=10$. Figure 2 presents the plots of power values against the departure from zero of the mean vector $\boldsymbol{\mu}=c \mathbf{1}_{p}$ with increasing $c$-values for relatively high dimension $p=50$ with a sample size $n \leq p$. The following two empirical conclusions can be summarized.

(1) The GF-test generally controlls thetype I error rates very well and shows fair effectiveness in testing the mean for the selected distributions in $E C D_{p}\left(\boldsymbol{\mu}, \sigma^{2} \boldsymbol{I}_{p}\right)$;

(2) The GF-test seems to be nonsensitive to the increase of the sample size. The power plots show that even the sample size is doubled, the power does not increase substantially. This implies that the $G F$-test may still have good power performance in the case of high dimension with a small sample size;

(3) The $G F$-test is applicable to the case of $n \leq p$. Classical tests for parametric hypotheses usually require $n>p$, for example, the Hotelling $T^{2}$-test for the multivariate normal mean. The power plots for the case of $n \leq p$ in Figure 2 show the effectiveness of the $G F$-test for the case of high dimension with a small sample size. For example, the power performance for $n=p / 2(p=50)$ is quite close to the case of $n=p=50$. The $G F$-test has an exact null distribution regardless of the sample size. This property makes the $G F$-test applicable for the case of any sample size. Therefore, the $G F$-test is especially applicable to the case of high dimension with a small sample size in real problems. 

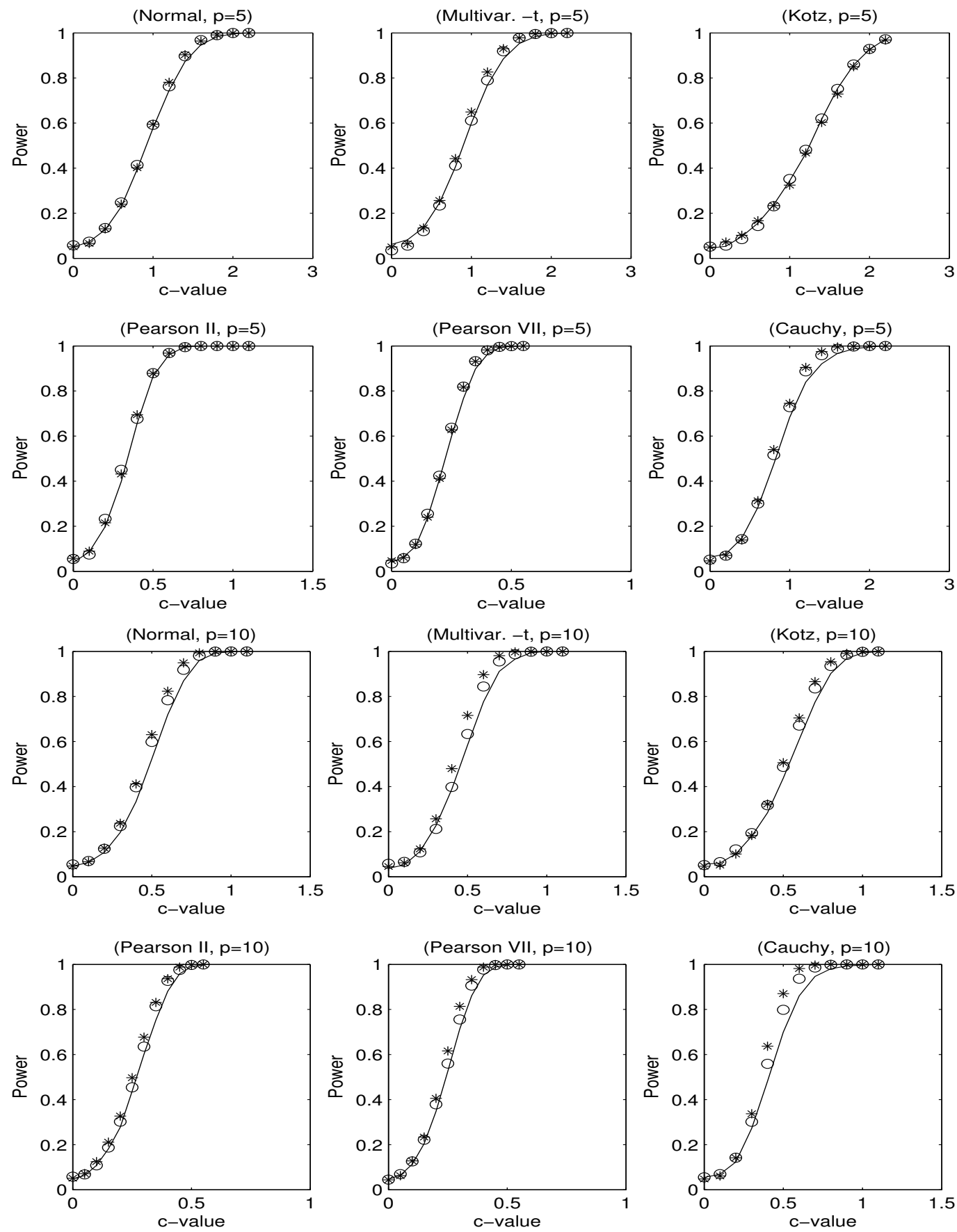

Figure 1. Power performance illustration for $\operatorname{six} \operatorname{ECD}\left(\mu, \sigma^{2} \boldsymbol{I}_{p}\right)(p=5$ and $p=10)$ : the real line stands for sample size $n=25$; the plot with "o" for sample size $n=50$; and the plot with "*" for sample size $n=100$. Mean vector $\boldsymbol{\mu}=c \mathbf{1}_{p}$.

\section{An Illustrative Example}

The value-weighted New York Stock Exchange return data are available from the Center for Research in Security Prices (CRSP) at the University of Chicago. We choose a partial data set (available from the authors upon request) that contains the market monthly returns between the year of 1966 and 1975 (120 months) from a portfolio with 32 stocks. First, we run the CAPM (capital asset pricing model, see 
[3] and [4]) to get the monthly risk-adjusted returns. These risk-adjusted returns constitute a sample with a sample size $n=120$ and dimension $p=32$. Second, we run a 2-factor analysis model to get another set of monthly risk-adjusted returns. These risk-adjusted returns also constitute a sample with a sample size $n=120$ and dimension $p=32$. Now we have two populations and two sets of samples:
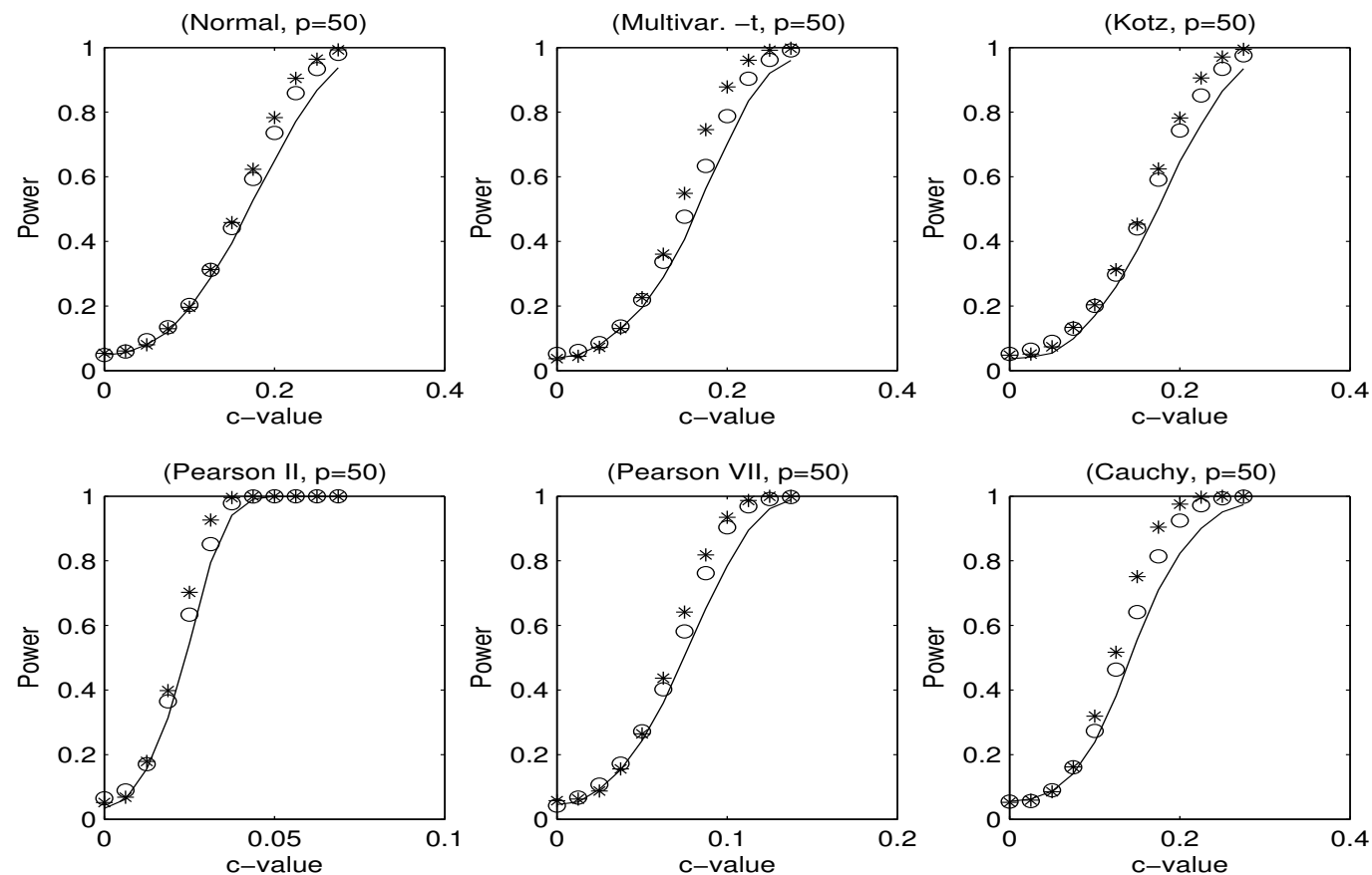

Figure 2. Power performance illustration for $\operatorname{six} \operatorname{ECD}\left(\mu, \sigma^{2} \boldsymbol{I}_{p}\right)(p=50)$ : the real line stands for sample size $n=25$; the plot with "o" for sample size $n=50$; and the plot with "*" for sample size $n=100$. Mean vector $\boldsymbol{\mu}=c \mathbf{1}_{p}$.

(1) Population \#1: risk-adjusted returns computed by CAPM, we have sample \#1 with $n=120$ and $p=32$;

(2) Population \#2: risk-adjusted returns computed by a 2 -factor model, we have sample \#2 with $n=120$ and $p=32$.

In the theory of CAPM, an investment portfolio is said to be efficient if it satisfies two conditions: 1 ) it reaches the smallest possible variance given its expected return; and 2) it reaches the largest possible expected return given its variance. The verification of these two conditions is finally reduced to testing the null hypothesis that the intercept parameter in the CAPM is equal to zero (vector) versus the alternative hypothesis that the intercept parameter in the CAPM is not equal zero. In the CAPM, the observations are the risk-adjusted returns. The purpose of constructing populations \#1 and \#2 is to double check the efficiency of the portfolio consisting of 32 stocks. Assuming population \#1 has an $E C D\left(\boldsymbol{\mu}_{1}, \sigma_{1}^{2} \boldsymbol{I}_{p}\right)$ and population \#2 has an $E C D\left(\boldsymbol{\mu}_{2}, \sigma_{2}^{2} \boldsymbol{I}_{p}\right)$, we set up the hypotheses:

$$
H_{0}: \boldsymbol{\mu}_{1}=\mathbf{0}, \quad \text { versus } \quad H_{1}: \boldsymbol{\mu}_{1} \neq \mathbf{0},
$$

and

$$
H_{0}: \boldsymbol{\mu}_{2}=\mathbf{0}, \quad \text { versus } \quad H_{1}: \boldsymbol{\mu}_{2} \neq \mathbf{0}
$$

The $p$-values of the $G F$-tests are 0.0000 and 0.0039 for hypotheses (9) and (10), respectively. The null hypotheses in (9) and (10) rejected at level 0.01. It is concluded that the two portfolios can be considered inefficient during the 10 years 1966-1975. 


\section{Concluding Remarks}

The $G F$-test in this paper provides a way to test the mean of some subfamilies of ECD under i.i.d. samples without assuming existance of the probability density function for the population. This is substantially different from many existing approaches to constructing parametric tests. The construction of the $G F$-test in Theorem 1 shows that the test is applicable for any dimension regardless of any sample size. This makes the $G F$-test particularly suitable for the case of high dimension with a small sample size. The Monte Carlo study supports this assertion. The real-data example illustrates possible applications of the $G F$-test in high-dimensional data analysis where the normal assumption may not be appropriate and the number of observations may be very limited due to high cost or difficulty in obtaining data. The $G F$-test is applicable for all population distributions in $E C D_{p}\left(\boldsymbol{\mu}, \sigma^{2} \boldsymbol{I}_{p}\right)$. This includes the normal distribution $N_{p}\left(\boldsymbol{\mu}, \sigma^{2} \boldsymbol{I}_{p}\right)$ as a special case. Therefore, the $G F$-test in this paper could find more applications in some areas like medical research where it is common to have data of high dimension with a small sample size and the normal assumption may be violated.

\section{References}

1. K. T. Fang, S. Kotz and K. W. Ng, Symmetric Multivariate and Related Distributions, Chapman and Hall, London and New York, 1990.

2. K. T. Fang and Y. Zhang, Generalized Multivariate Analysis, Springer-Verlag and Science Press, Berlin/Beijing, 1990.

3. M. R. Gibbons, S. Ross and J. Shanken, "A test of efficiency of a given portfolio," Econometrica, vol. 57, pp. 1121-1152, 1989.

4. C. A. MacKinlay, "On multivariate tests of the CAPM," Journal of Financial Economics, vol. 18, pp. 341-372, 1987. 